\title{
Sewing Polyakov Amplitudes I: Sewing at a Fixed Conformal Structure
}

\author{
Steven Carlip, ${ }^{1 \star}$ Minot Clements, ${ }^{2 \star \star}$ Stephen DellaPietra ${ }^{1,3}$ and Vincent \\ DellaPietra ${ }^{3}$ \\ ${ }^{1}$ Institute for Advanced Study, Princeton, NJ 08540, USA \\ ${ }^{2}$ University of Washington, Seattle, WA 98195, USA \\ ${ }^{3}$ IBM Watson Research Center, Yorktown Heights, NY 10598, USA
}

\begin{abstract}
We consider the problem of reconstructing the correlation functions of a conformal field theory on a surface $\Sigma$ from the correlation functions on a surface $\Sigma^{\prime}$ obtained from $\Sigma$ by cutting along a closed curve. We show that under quite general conditions, the correlation functions on the cut surface can be "sewn" by integrating over appropriate boundary values of the fields.
\end{abstract}

\section{Introduction}

In quantum field theory, one ordinarily begins with a Lagrangian and derives a perturbation expansion and Feynman rules. In string theory, this process has been reversed. We have an elegant set of Feynman rules, given by the Polyakov path integral; but despite numerous attempts to write down a field theory of closed strings, a generally accepted formulation does not yet exist. It is thus natural to ask whether information about a field theory can be obtained from the Polyakov path integral. ${ }^{1}$ In particular, we may ask whether it is possible to derive higher order terms in the perturbation expansion-path integrals over higher genus surfaces - from lower order terms. This is the "sewing" problem.

The sewing problem consists of two distinct elements. The first may be called sewing at a fixed conformal structure. We start with a string world sheet with a given conformal structure, and cut it along a curve to form a new (possibly disconnected) world sheet, which inherits a conformal structure from the original surface. We can then attempt to reconstruct the Polyakov measure on the original world sheet from the measure on the cut surface. If it is possible, such a reconstruction will imply strong relationships between determinants and Greens functions on the two surfaces. More generally, we may start with an arbitrary conformal field theory, and attempt to reconstruct the partition function and

\footnotetext{
* Supported in part by DOE grant DE-AC02-76ERO2220

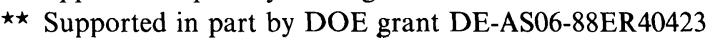

${ }^{1}$ The idea of using the Polyakov path integral to develop a string field theory was first discussed by Tseytlin [1]
} 
correlation functions on the original surface from corresponding quantities on the cut surface.

The second element of the sewing problem is the sewing of transition amplitudes. We now start with the full (off-shell) Polyakov amplitude, already integrated over moduli, for a surface with two or more boundary curves; our goal is to construct the amplitude for the surface obtained by identifying a pair of boundaries. This task is more difficult; the identification map between the two boundary curves is no longer uniquely specified, and the relationship between the moduli spaces of the cut and sewn surfaces must be understood. Nevertheless, if a closed string field theory exists, it should be possible to find a procedure for sewing amplitudes.

In this paper, we address the first question of sewing at fixed conformal structure. We demonstrate that it is possible to sew arbitrary correlation functions for a wide variety of conformal field theories by functional integration over boundary values of the fields. Sonoda [2] has given an indirect argument for this result; we take the more direct approach of explicitly proving the required relationships among determinants and Greens functions. A subsequent paper will discuss the second aspect of sewing, the sewing of Polyakov amplitudes. A preliminary announcement of this work has appeared in [3].

\section{Sewing at Fixed Conformal Structure}

We start with a Riemann surface $\Sigma$, and cut along a curve $C$ to form a new, possibly disconnected surface $\Sigma^{\prime}$ (see Fig. 1). Our goal is to show that correlation functions for a conformal field theory on $\Sigma$ can be obtained from the corresponding correlation functions on $\Sigma^{\prime}$ by functional integration over the boundary values of the fields on $C$ : schematically,

$$
\langle\phi \cdots \phi\rangle_{\Sigma}=\int\left[\left.d \phi\right|_{C}\right]\langle\phi \cdots \phi\rangle_{\Sigma^{\prime}} .
$$

In one sense, this relation is obvious. Correlation functions on $\Sigma$ can be obtained
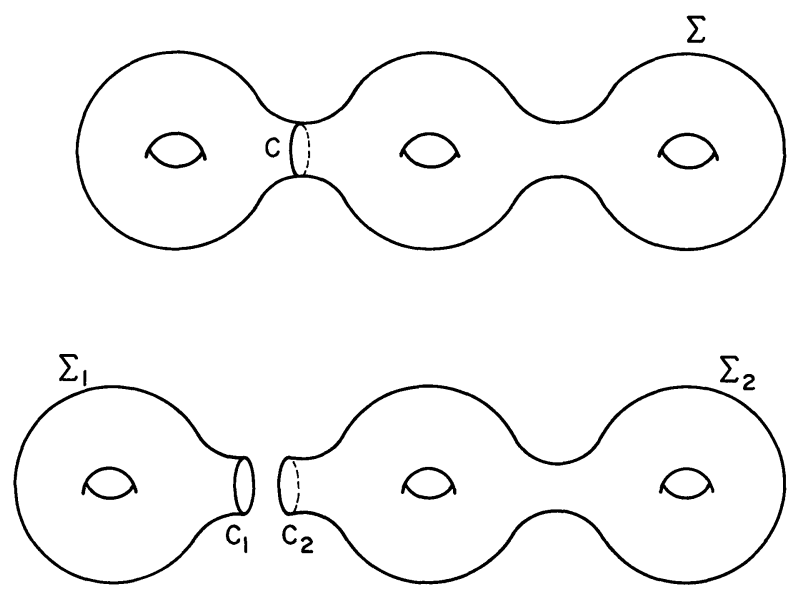

Fig. 1. The surface $\Sigma$ is cut along the curve $C$ to produce a new surface $\Sigma^{\prime}$ with boundaries $C_{1}$ and $C_{2}$ 
from a path integral, and it should be possible to evaluate such an integral by first integrating over fields with specified boundary values on $C$, and then integrating over those boundary values. On the other hand, this sewing process implies some very unobvious relations between determinants and Green functions on $\Sigma$ and $\Sigma^{\prime}$, and a proof which does not depend directly on the path integral is clearly desirable. This is especially true when there are zero modes present, since even the definition of the path integral then becomes somewhat subtle.

To understand what is involved, consider the simplest case of a free scalar field $X$, with an action

$$
S=\frac{1}{2} \int \partial X \wedge \bar{\partial} X .
$$

Suppose for simplicity that $\Sigma$ is closed, so $\Sigma^{\prime}$ has two boundary components $C_{1}$ and $C_{2}$ (see Fig. 1), which are identified to form the curve $C$ on $\Sigma$. The partition function is

$$
Z_{\Sigma^{\prime}}\left[\tilde{X}_{i}\right]=\int_{\left.X\right|_{C_{i}}=\tilde{X}_{i}}[d X] e^{-S[X]},
$$

where the $\tilde{X}_{i}(i=1,2)$ are the specified boundary values of $X$ on $C_{i}$.

The techniques for evaluating this integral are standard. We split $X$ into a classical piece $\bar{X}$, satisfying $\Delta \bar{X}=0,\left.\bar{X}\right|_{C_{t}}=\widetilde{X}_{i}$, and a fluctuation $X^{\prime}$ which vanishes on $C_{i}, \bar{X}$ and $X^{\prime}$ decouple in the action $S$, and the $X^{\prime}$ integral is Gaussian; hence

$$
Z_{\Sigma^{\prime}}\left[\tilde{X}_{i}\right]=\left(\operatorname{det} \Delta_{\Sigma^{\prime}}\right)^{-1 / 2} e^{-\bar{S}\left[\tilde{X}_{i}\right]},
$$

where the determinant is evaluated for Dirichlet boundary conditions and

$$
\bar{S}\left[\tilde{X}_{i}\right]=S[\bar{X}]=\frac{1}{2} \sum_{i j} \int_{C_{i}} d x \int_{C_{j}} d x^{\prime} \tilde{X}_{i}(x) \partial_{n_{i}} \partial_{n_{j}} G\left(x, x^{\prime}\right) \tilde{X}_{j}\left(x^{\prime}\right) .
$$

Here $G\left(x, x^{\prime}\right)$ is the Dirichlet Greens function, and $\partial_{n}$ denotes the normal derivative; $\bar{S}\left[\tilde{X}_{i}\right]$ is the classical action for the boundary data $\tilde{X}_{i}$.

For sewing to hold, we must have

$$
Z_{\Sigma}=\int[d \tilde{X}] Z_{\Sigma^{\prime}}[\tilde{X}, \tilde{X}] .
$$

By (2.3), this will be the case if

$$
\operatorname{det} \Delta_{\Sigma}=\operatorname{det} \Delta_{\Sigma^{\prime}} \operatorname{det}\left(\sum_{i j} \partial_{n_{i}} \partial_{n_{j^{\prime}}} G\right) .
$$

Such a relation between determinants is far from obvious, but as we shall demonstrate below, it is true.

To gain some feeling for the problem, let us first explicitly verify the one-dimensional version of (2.6) for a simple example. Let $\Sigma$ be an interval $[0, l]$, and cut at $l_{1}$ to form a pair of intervals $\Sigma^{\prime}=\left[0, l_{1}\right] \cup\left[l_{1}, l\right]$. Choosing Dirichlet boundary conditions $X(0)=X(l)=0$ and $X\left(l_{1}\right)=\tilde{X}$ for $\Sigma^{\prime}$, we find the classical solution

$$
\bar{X}(x)=\left\{\begin{array}{ll}
\tilde{X} \frac{x}{l_{1}} & x \leqq l_{1} \\
\tilde{X} \frac{l-x}{l-l_{1}} & x \geqq l_{1}
\end{array},\right.
$$


giving

$$
\bar{S}[\tilde{X}]=\tilde{X}^{2}\left(\frac{1}{l_{1}}+\frac{1}{l-l_{1}}\right) .
$$

The determinants in (2.6) are

$$
\operatorname{det} \Delta_{[0, l]}=\left(\prod_{n>0} \frac{n^{2} \pi^{2}}{l^{2}}\right) .
$$

Performing the integral in (2.5), we find that (2.6) now becomes

$$
\left(\prod_{n>0} \frac{n^{2} \pi^{2}}{l^{2}}\right)=\left(\prod_{n_{1}>0} \frac{n_{1}^{2} \pi^{2}}{l_{1}^{2}}\right)\left(\prod_{n_{2}>0} \frac{n_{2}^{2} \pi^{2}}{\left(l-l_{1}\right)^{2}}\right) \frac{l}{l_{1}\left(l-l_{1}\right)} .
$$

The infinite products may be regulated using zeta functions:

$$
\prod_{n>0} \frac{n^{2} \pi^{2}}{l^{2}}=\exp \sum_{n>0} \ln \frac{n^{2} \pi^{2}}{l^{2}}=\exp \lim _{s \rightarrow 0}\left\{-\frac{d}{d s}\left[\left(\frac{\pi^{2}}{l^{2}}\right)^{-s} \zeta(2 s)\right]\right\}=2 l,
$$

where we have used $\zeta(0)=-\frac{1}{2}$ and $\zeta^{\prime}(0)=-\frac{1}{2} \ln 2 \pi$. Hence $(2.10)$ is satisfied up to factors of 2 which can be absorbed in the functional measure. Our aim is to generalize this result to two dimensions with arbitrary topologies, operators, and boundary conditions.

The kernel $\partial_{n} \partial_{n^{\prime}} G\left(x, x^{\prime}\right)$ in (2.4) and (2.6) has an interpretation which will be useful later. Imagine specifying $\tilde{X}$ on $\partial \Sigma^{\prime}$, extending this Dirichlet boundary data harmonically to the interior of $\Sigma^{\prime}$, and then calculating the corresponding Neumann data $\partial_{n} X$ for the harmonic extension. It is easy to see that $\partial_{n} \partial_{n^{\prime}} G\left(x, x^{\prime}\right)$ maps the original Dirichlet data to the corresponding Neumann data. Forman [5] calls such a map between different boundary data a Poisson map; we will see that such a map appears quite generally.

\section{Action, Boundary Conditions, and Greens Functions}

Rather than trying to show that the relation (2.6) directly, we will work in a more general setting. We continue to assume that $\Sigma$ is closed (the generalization to bordered surfaces is not hard), but now consider a general Gaussian conformal field theory with an action

$$
S=\int_{\Sigma} b L c
$$

where $b \in V^{-}$and $c \in V^{+}$are sections of vector bundles over $\Sigma$ and $L: V^{+} \rightarrow V^{-}$is a linear differential operator of order $n$. In this section, we also assume that there are no zero modes, i.e., that $L$ has no kernel or cokernel; this assumption will be relaxed in Sect. 6. The notation of (3.1) suggests a standard $b-c$ system, but we do not restrict ourselves to that case. For instance, we can take $b=c=X, L=-\frac{1}{2} \Delta$, and recover the scalar action (2.1), up to a boundary term which will be discussed below.

This general setting will simplify and broaden our proofs, but at the cost of some extra notation. The main purpose of this section is to introduce notation; the proof of (2.6) will be carried out in Sect. 4, with successive generalizations 
in Sects. 5-7. To minimize confusion, we will frequently refer to the scalar field as an illustration.

As in the scalar case, we must first specify boundary data for $b$ and $c$. We begin with $c$. Let $\mathscr{C}^{+}$denote the Cauchy data for $c$ (i.e., its boundary value and its first $n-1$ normal derivatives), and let $\pi: V^{+} \rightarrow \mathscr{C}^{+}$be the projection of $c$ onto its Cauchy data. A suitable set of boundary data for $c$ consists of half of its Cauchy data (e.g., Dirichlet or Neumann data for a scalar field, or the positive frequency part of a spinor field for which $L$ is a first order operator). We label a choice of boundary data by a capital letter such as $A$, and define a new projection $\pi_{A}: V^{+} \rightarrow \mathscr{C}^{+}$of $c$ onto its $A$ boundary data.

More precisely, we can view $A$ as a projection $A: \mathscr{C}^{+} \rightarrow \mathscr{C}^{+}$from the Cauchy data to the $A$ boundary data; then $\pi_{A}=A \circ \pi$. Observe that $1-A: \mathscr{C}^{+} \rightarrow \mathscr{C}^{+}$is also a projection onto half of the Cauchy data, so $\pi_{1-A}$ defines another set of boundary data. For the scalar field, for instance, $\mathscr{C}=\left\{\left.\left(X, \partial_{n} X\right)\right|_{C}\right\}$, Dirichlet data is given by the projection $A:\left.\left(X, \partial_{n} X\right)\right|_{C} \mapsto\left(\left.X\right|_{C}, 0\right)$, and Neumann data is given by the projection $1-A$.

For a given choice of boundary data $A$, the corresponding boundary condition on $L$ is given by restricting $L$ to fields lying in $\operatorname{ker} \pi_{A}$. In the scalar case, for instance, Dirichlet boundary conditions correspond to restricting the Laplacian $\Delta$ to fields which vanish at the boundary. For a scalar field, it is well known that a Greens function for Dirichlet boundary conditions can be used to determine $X$ from its Dirichlet boundary data; a similar statement is true in general. We denote by $L_{A}$ the operator obtained by restricting $L$ to $\operatorname{ker} \pi_{A}$.

Given a choice of boundary data for $c$, we must next determine suitable adjoint boundary data for $b$. We again define a projection $\pi: V^{-} \rightarrow \mathscr{C}^{-}$of $b$ onto its Cauchy data $\mathscr{C}^{-}$, and consider the bilinear functional

$$
\int_{\Sigma^{\prime}}\left(b L c-L^{\dagger} b c\right)=W(\pi b, \pi c) .
$$

DeWitt [4] calls $W$ the Wronskian. We can now define the adjoint boundary data $A^{\dagger}$ by the requirement that

$$
W(\pi b, \pi c)=W\left(\pi b, \pi_{A} c\right)+W\left(\pi_{A^{+}} b, \pi c\right),
$$

that is,

$$
W\left(\pi_{A^{+}} b, \pi c\right)=W\left(\pi b, \pi_{1-A} c\right) .
$$

The data $A^{\dagger}$ are adjoint to $A$ in the sense that $\left(L^{\dagger}\right)_{A^{\dagger}}=\left(L_{A}\right)^{\dagger}$. Observe that if the fields $b$ and $c$ are identical, we must choose self-adjoint boundary data, $A=A^{\dagger}$. For a scalar field, for example, $W(\pi X, \pi Y)=\int_{C}\left(-X \partial_{n} Y+Y \partial_{n} X\right)$, and both Dirichlet and Neumann data are self-adjoint.

As in the scalar case, we will need a Greens function for $L$. We assume that $L_{A}$ is invertible, and define the Greens function $G_{A}: V^{-} \rightarrow V^{+}$with $A$ boundary conditions by

$$
L G_{A}=1,\left.\quad G_{A} L\right|_{\mathrm{ker} \pi_{A}}=\left.1\right|_{\mathrm{ker} \pi_{A}}, \quad \pi_{A} G_{A}=0 .
$$

Given such a Greens function, we can solve the boundary value problem

$$
L c=0 \quad \text { with } \quad \pi_{A} c=\tilde{c} .
$$


We will refer to the solution of such a boundary value problem as the extension by $L$ of the boundary data. We thus have a map $P_{A}: \operatorname{Im} \pi_{A} \rightarrow V^{+}$which maps the boundary data $\tilde{c}$ to its extension by $L ; P_{A}$ is determined by the requirement that

$$
L P_{A}=0, \quad \pi_{A} P_{A}=1 .
$$

The solution of this equation is

$$
P_{A} \pi_{A}=1-G_{A} L,
$$

as may be verified by acting on the left with $L$ and with $\pi_{A}$. For a scalar field $X$, (3.7) is the statement that $\int d x^{\prime} G\left(x, x^{\prime}\right) \Delta X\left(x^{\prime}\right)=(X-\bar{X})(x)$, where $\bar{X}$ is the harmonic extension of $\left.X\right|_{C}$; this relation follows from Greens theorem.

Having introduced the required notation, let us return to the action (3.1). As Birmingham and Torre [6] first pointed out for the bosonic string ghost action, (3.1) is inadequate when $\Sigma^{\prime}$ has a boundary: solutions of the equations of motion are not true extrema of $S$. Indeed, write $b=\bar{b}+b^{\prime}, c=\bar{c}+c^{\prime}$, where $\bar{b}$ and $\bar{c}$ are classical fields $\left(L \bar{c}=0=L^{\dagger} \bar{b}\right)$ and $b^{\prime}$ and $c^{\prime}$ are fluctuations with vanishing boundary data $\left(\pi_{A} c^{\prime}=0=\pi_{A^{+}} b^{\prime}\right)$. Then it is easy to check that $S[b, c]=S\left[b^{\prime}, c^{\prime}\right]+W\left(\pi \bar{b}, \pi c^{\prime}\right)$, and the classical fields fail to decouple from the fluctuations.

The presence of the cross term makes it impossible to use standard methods to evaluate the path integral. To eliminate it, we must add to the action (3.1) a boundary contribution of the form

$$
S_{1}=-W\left(\pi_{A^{+}} b, \pi_{1-A} c\right) \text {. }
$$

We then find that

$$
\left(S+S_{1}\right)[b, c]=S\left[b^{\prime}, c^{\prime}\right]-W\left(\pi_{A^{+}} \bar{b}, \pi_{1-A} \bar{c}\right),
$$

so the classical fields and fluctuations now decouple. For the scalar field with Dirichlet boundary data, for example, $S=-\frac{1}{2} \int_{\Sigma} X \Delta X$, while $S_{1}=\frac{1}{2} \int_{\partial \Sigma} X \partial_{n} X$, so
$S+S_{1}$ gives the standard action (2.1).

The extension map $P_{A}$ allows us to write $\bar{b}$ and $\bar{c}$ in (3.9) in terms of their boundary values $\tilde{b}=\pi_{A^{+}} b$ and $\tilde{c}=\pi_{A} c$. We find that

$$
\left(S+S_{1}\right)[b, c]=S\left[b^{\prime}, c^{\prime}\right]-W\left(\tilde{b}, \pi_{1-A} P_{A} \tilde{c}\right) .
$$

The term involving $W$ is the generalization of the classical action (2.4) for the scalar field. The map $\pi_{1-A} P_{A}$ is a Poisson map: it is the projection onto $1-A$ boundary data of the extension by $L$ of $A$ boundary data. If we denote the Poisson map between two sets of boundary data $A$ and $B$ by

$$
\phi_{B, A}=\pi_{B} P_{A},
$$

we find that

$$
Z_{\Sigma^{\prime}}[\tilde{b}, \tilde{c}]=\int[d b][d c] e^{-\left(S+S_{1}\right)[b, c]}=\left(\operatorname{det} L_{\Sigma^{\prime}}\right)^{ \pm 1} \exp \left\{W\left(\tilde{b}, \phi_{1-A, A} \tilde{c}\right)\right\},
$$

where the sign in the exponent of the determinant is negative if $b$ and $c$ are commuting, positive if they are anticommuting.

Equation (3.12) is the generalization to Gaussian conformal theories of (2.3) for the scalar field. To find the generalization of (2.6), we must integrate the partition 
function over boundary values of $b$ and $c$. As in the scalar case, there is a contribution from each of the boundary components $C_{1}$ and $C_{2}$. We can write the projection $\pi_{A}$ as

$$
\pi_{A}=\left(\begin{array}{c}
\pi_{A}^{1} \\
\pi_{A}^{2}
\end{array}\right)
$$

where $\pi_{A}^{i}$ projects onto the $A$ boundary data on the boundary $C_{i}$. Similarly, since $L$ is linear, the extension map $P_{A}$ takes the form

$$
P_{A}=\left(\begin{array}{ll}
P_{A}^{1} & P_{A}^{2}
\end{array}\right)
$$

where $P_{A}^{i}$ extends the boundary data on $C_{i}$ with zero boundary data on $C_{j \neq i}$, that is, $L P_{A}^{i}=0, \pi_{A}^{i} P_{A}^{j}=\delta_{i j}$. (Note that if $i$ and $j$ label boundaries on two distinct surfaces, then $\pi_{B}^{i} P_{A}^{j}=0$ for any choice of boundary data $A$ and $B$.)

The functional integral for sewing is to be evaluated with the boundary values on $C_{1}$ and $C_{2}$ set equal; it is thus

$$
\underset{\substack{\tilde{b}_{1}=\tilde{c}_{2} \\ \tilde{c}_{1}=\tilde{c}_{2}}}{ }[d \tilde{b}][d \tilde{c}] Z_{\Sigma^{\prime}}[\tilde{b}, \tilde{c}]=\left(\operatorname{det} L_{\Sigma^{\prime}}\right)^{ \pm 1} \int[d \tilde{b}][d \tilde{c}] \exp \left\{\sum_{i j} W\left(\tilde{b}, \phi_{1}^{i j}-A, A \tilde{c}\right)\right\},
$$

where

$$
\phi_{1-A, A}^{i j}=\pi_{1-A}^{i} P_{A}^{j} .
$$

Now observe that $W$ is a bilinear form, so the integral in (3.15) is Gaussian; up to a constant factor, it will equal $\left(\operatorname{det} \sum_{i j} \phi_{1-A, A}^{i j}\right)^{ \pm 1}$. The desired generalization of $(2.6)$ is thus

$$
\operatorname{det} L_{\Sigma}=\operatorname{det} L_{\Sigma^{\prime}} \operatorname{det}\left(\sum_{i j} \phi_{1-A, A}^{i j}\right) .
$$

If we can prove (3.17), we will shown that sewing holds for the partition function $Z_{\Sigma^{\prime}}$. By considering the generating functional for correlation functions, we will see below that this also suffices to show sewing for arbitrary correlation functions.

\section{Sewing without Zero Modes}

We now turn to the proof of (3.17). Our approach is to examine arbitrary variations of $\operatorname{det} L$ and $\operatorname{det} \phi$ to show that an infinitesimal version of (3.17) holds; the finite version will then be true up to an overall integration constant. The proof is complicated by the fact that (3.17) compares determinants on two different surfaces. We can avoid this difficulty, however, by considering the determinant on $\Sigma$ to be a determinant on the cut surface $\Sigma^{\prime}$, but with "sewing boundary conditions": we require that the entire Cauchy data of $b$ and $c$ be continuous across $C$. There is a one-to-one correspondence between the eigenfunctions of $L$ on $\Sigma^{\prime}$ with such sewing boundary conditions and the eigenfunctions of $L$ on $\Sigma$.

Some care is needed here, however, as may be seen most easily in the case of the scalar field. It is certainly true that eigenfunctions of $\Delta$ on the sewn surface 
will have equal Dirichlet data on $C$. If the normal derivative is defined in terms of an outward-pointing normal vector, however, we must have $\left.\partial_{n} X\right|_{C_{1}}=-\left.\partial_{n} X\right|_{C_{2}}$, since the normal changes direction between the two boundaries. Hence if $A$ is the projection onto Dirichlet data, we need $\pi_{A}^{1}=\pi_{A}^{2}$, but $\pi_{1-A}^{1}=-\pi_{1-A}^{2}$.

More generally, we must specify an identification of the Cauchy data on the two boundary curves being sewn before we can describe sewing boundary conditions. We have already implicitly made such an identification in the previous section, where we asserted that the functional integral was to be performed over equal boundary values $\tilde{b}_{1}=\widetilde{b}_{2}, \tilde{c}_{1}=\tilde{c}_{2}$, i.e., $\pi_{A}^{1}=\pi_{A}^{2}, \pi_{A^{\dagger}}^{1}=\pi_{A^{+}}^{2}$. It remains for us to find the corresponding relations for $\pi_{1-A}$ and $\pi_{1-A^{+}}$.

To do so, note that for sewing boundary conditions, the total Wronskian $W(\pi b, \pi c)$ must vanish. Indeed, $W$ is a boundary term arising from partial integration of $L$ in equation (3.2), and there can be no such boundary term if there is no boundary. But for $\tilde{b}_{1}=\tilde{b}_{2}, \tilde{c}_{1}=\tilde{c}_{2}$,

$$
W(\pi b, \pi c)=W\left(\pi_{1-A^{+}}^{1} b+\pi_{1-A^{+}}^{2} b, 2 \tilde{c}\right)+W\left(2 \tilde{b}, \pi_{1-A}^{1} c+\pi_{1-A}^{2} c\right),
$$

which will vanish for arbitrary $\tilde{b}$ and $\tilde{c}$ only if $\pi_{1-A^{\dagger}}^{1} b=-\pi_{1-A^{+}}^{2} b$ and $\pi_{1-A}^{1} c=-\pi_{1-A}^{2} c$. The extra minus sign which we found for the scalar field is thus a general phenomenon.

To proceed further, let $A$ and $B$ be two arbitrary choices of boundary data. We first note that

$$
\phi_{A, B}^{-1}=\phi_{B, A} .
$$

Indeed, by (3.7), $\phi_{B, A} \phi_{A, B}=\pi_{B} P_{A} \pi_{A} P_{B}=\pi_{B}\left(1-G_{A} L\right) P_{B}=\pi_{B} P_{B}=1$. Hence an arbitrary variation $\delta$ of $\operatorname{det} \phi_{B, A}$ is

$$
\delta \log \operatorname{det} \phi_{B, A}=\operatorname{Tr} \phi_{B, A}^{-1} \delta \phi_{B, A}=\operatorname{Tr} \pi_{A} P_{B} \pi_{B} \delta P_{A} .
$$

We next observe that $\delta\left(L P_{A}\right)=0=(\delta L) P_{A}+L\left(\delta P_{A}\right)$; acting on the left with $G_{A}$, and noting that $\delta P_{A}$ is in the kernel of $\pi_{A}$ (as can be seen by varying the equation $\left.\pi_{A} P_{A}=1\right)$, we see that

$$
\delta P_{A}=-G_{A} \delta L P_{A} .
$$

We also note that $G_{A}$ is the inverse of $L_{A}$, so

$$
\delta \log \operatorname{det} L_{A}=\operatorname{Tr} G_{A} \delta L .
$$

Combining (4.3), (4.4), and (4.5), we find that

$$
\begin{aligned}
\delta \log \left(\operatorname{det} \phi_{B, A} \operatorname{det} L_{A}\right) & =\operatorname{Tr}\left[G_{A} \delta L-\pi_{A} P_{B} \pi_{B} G_{A} \delta L P_{A}\right] \\
& =\operatorname{Tr}\left[G_{A}-P_{A} \pi_{A} P_{B} \pi_{B} G_{A}\right] \delta L \\
& =\operatorname{Tr}\left[G_{A}-\left(1-G_{A} L\right)\left(1-G_{B} L\right) G_{A}\right] \delta L \\
& =\operatorname{Tr} G_{B} \delta L=\delta \log \operatorname{det} L_{B},
\end{aligned}
$$

where we have used cyclicity of the trace in the second line. Hence, up to a constant factor,

$$
\operatorname{det} L_{B}=\operatorname{det} \phi_{B, A} \operatorname{det} L_{A} \text {, }
$$


a result first shown in a more special context by Forman [5]. This relation is the key to our proof of sewing.

We now apply this general result to relate $A$ boundary conditions to sewing boundary conditions. As discussed above, sewing boundary conditions require that $\pi_{A}^{1}=\pi_{A}^{2}$ and $\pi_{1-A}^{1}=-\pi_{1-A}^{2}$. This will be the case if we define our boundary data by the projection

$$
\pi_{B}=\left(\begin{array}{c}
\pi_{A}^{1}-\pi_{A}^{2} \\
\pi_{1-A}^{1}+\pi_{1-A}^{2}
\end{array}\right) .
$$

$L_{B}$ then corresponds to $L$ on $\Sigma$, while $L_{A}$ is $L$ on $\Sigma^{\prime}$ with $A$ boundary conditions, and the partition function on $\Sigma$ is equivalent to the partition function on $\Sigma^{\prime}$ with vanishing $B$ boundary data. The Poisson map $\phi_{B, A}$ is now

$$
\begin{aligned}
\phi_{B, A}=\pi_{B} P_{A} & =\left(\begin{array}{cc}
\left(\pi_{A}^{1}-\pi_{A}^{2}\right) P_{A}^{1} & \left(\pi_{A}^{1}-\pi_{A}^{2}\right) P_{A}^{2} \\
\left(\pi_{1-A}^{1}+\pi_{1-A}^{2}\right) P_{A}^{1} & \left(\pi_{1-A}^{1}+\pi_{1-A}^{2}\right) P_{A}^{2}
\end{array}\right) \\
& =\left(\begin{array}{cc}
1 & -1 \\
\phi_{1-A, A}^{11}+\phi_{1-A, A}^{21} & \phi_{1-A, A}^{12}+\phi_{1-A, A}^{22}
\end{array}\right),
\end{aligned}
$$

so

$$
\operatorname{det} \phi_{B, A}=\operatorname{det} \sum_{i j} \phi_{1-A, A}^{i j}
$$

Comparing (4.7) and (4.10), we have succeeded in proving the sewing relation (3.17).

\section{Sewing Correlation Functions}

We have now demonstrated sewing for the partition function. Let us next investigate the sewing of arbitrary correlation functions. We do so by adding sources for $b$ and $c$ and studying the resulting generating functional.

Let

$$
S_{2}=\int_{\Sigma} b J+K c
$$

where $J \in V^{-}$and $K \in V^{+}$are sources for $b$ and $c$. Any correlation function on $\Sigma^{\prime}$ can be obtained from the generating functional

$$
Z_{\Sigma^{\prime}}[\tilde{b}, \tilde{c}, J, K]=\int[d b][d c] e^{-\left(\boldsymbol{S}+\boldsymbol{S}_{1}+\boldsymbol{S}_{2}\right)[b, c, J, K]}
$$

by functional differentiation with respect to $J$ and $K$. To evaluate $Z_{\Sigma^{\prime}}$, we again write $b=\bar{b}+b^{\prime}, c=\bar{c}+c^{\prime}$, where now

$$
\begin{aligned}
L \bar{c}+J & =0, \quad \pi_{A} c^{\prime}=0, \\
L^{\dagger} \bar{b}+K & =0, \quad \pi_{A^{\dagger}} b^{\prime}=0,
\end{aligned}
$$

which implies that

$$
\bar{c}=P_{A} \pi_{A} \bar{c}-G_{A} J, \quad \bar{b}=P_{A^{\dagger}} \pi_{A^{\dagger}} \bar{b}-G_{A^{\dagger}} K .
$$

A simple calculation shows that 


$$
\begin{aligned}
\left(S+S_{1}+S_{2}\right)[b, c, J, K]= & S\left[b^{\prime}, c^{\prime}\right]-W\left(\pi_{A^{\dagger}} \bar{b}, \phi_{1-A, A} \pi_{A} \bar{c}\right) \\
& +\int\left[\left(P_{A^{\dagger}} \pi_{A^{\dagger}} \bar{b}\right) J+K\left(P_{A} \pi_{A} \bar{c}\right)-\left(G_{A^{\dagger}} K\right) J\right],
\end{aligned}
$$

and thus (again denoting the boundaries of $\Sigma^{\prime}$ by $C_{1}$ and $C_{2}$ )

$$
\begin{aligned}
& Z_{\Sigma^{\prime}}[\tilde{b}, \tilde{c}, J, K] \\
& \quad=\left(\operatorname{det} L_{\Sigma^{\prime}}\right)^{ \pm 1} \exp \left\{W\left(\tilde{b}_{i}, \phi_{1-A, A}^{i j} \tilde{c}_{j}\right)-\int\left[\left(P_{A^{i}}^{i} \tilde{b}_{i}\right) J+K\left(P_{A}^{i} \tilde{c}_{i}\right)-\left(G_{A^{\dagger}} K\right) J\right]\right\} .
\end{aligned}
$$

To sew $C_{1}$ and $C_{2}$, we must again set $\tilde{b}_{1}=\tilde{b}_{2}$ and $\tilde{c}_{1}=\tilde{c}_{2}$ and integrate over boundary values. The integral is still Gaussian, but the exponent in (5.6) now contains terms linear in $\tilde{b}$ and $\tilde{c}$, so the integral no longer simply gives a determinant. As usual, however, we can eliminate the linear terms by shifting $\tilde{b}$ and $\tilde{c}$. Let us denote the shift in $\tilde{b}$ by $\widetilde{\beta}$, where $\widetilde{\beta}=\pi_{A^{+}}^{1} \beta=\pi_{A^{+}}^{2} \beta$ for a function $\beta$ which must be determined. Naturally, $\beta$ must have the same value on the two boundaries being sewn, so $\pi_{B^{\dagger}}^{1} \beta=0$, where $\pi_{B}$ is the projection onto sewing boundary conditions given by (4.8). The remaining component of $\pi_{B^{\dagger}}$ will appear shortly.

To determine the effect of the shift of $\tilde{b}$, we consider the first term in the exponent of (5.6). Now,

$$
\begin{aligned}
W\left(\pi_{A^{+}}^{i} \beta, \phi_{1-A, A}^{i j} \pi_{A}^{j} \bar{c}\right) & =W\left(\pi^{i} \beta, \pi_{1-A}^{i} P_{A}^{j} \pi_{A}^{j} \bar{c}\right) \\
& =W\left(\pi^{i} \beta,\left(\pi^{i} P_{A}^{j} \pi_{A}^{j}-\pi_{A}^{i} P_{A}^{j} \pi_{A}^{j}\right) \bar{c}\right) \\
& =-\int\left(L^{\dagger} \beta\right) P_{A}^{j} \pi_{A}^{j} \bar{c}-W\left(\pi_{1-A^{+}}^{i} \beta, \pi_{A}^{i} \bar{c}\right),
\end{aligned}
$$

where we have used the definitions (3.2) and (3.3) and the relations $\pi_{A}^{i} P_{A}^{j}=\delta_{i j}$ and $L P_{A}^{j}=0$. But for sewing, $\pi_{A}^{1} \bar{c}=\pi_{A}^{2} \bar{c}=\tilde{c}$, so the last term in (5.7) will vanish if $\pi_{1-A^{\dagger}}^{1} \beta+\pi_{1-A^{\dagger}}^{2} \beta=0$, i.e., $\pi_{B^{+}}^{2} \beta=0$. The term in (5.6) linear in $\tilde{c}$ will thus be eliminated by the shift of $\tilde{b}$ given by

$$
L^{\dagger} \beta=-K, \quad \pi_{B^{\dagger}} \beta=0 .
$$

Equation (5.8) is satisfied by $\beta=-G_{B^{\dagger}} K$, and the generating functional (5.6) is thus

$$
\begin{aligned}
Z_{\Sigma^{\prime}}[\tilde{b}, \tilde{c}, J, K]= & \left(\operatorname{det} L_{\Sigma^{\prime}}\right)^{ \pm 1} \exp \left\{W\left((\tilde{b}-\tilde{\beta}), \sum_{i j} \phi_{1-A, A^{i j}}^{i j}\right)\right. \\
& -\int\left[\left(P_{A^{+}}^{i} \pi_{A^{+}}^{i}(\tilde{b}-\widetilde{\beta}) J-\left(G_{A^{\dagger}} K\right) J\right]-\int\left(P_{A^{\dagger}}^{i} \pi_{A^{\dagger}}^{i} \tilde{\beta}\right) J\right\} .
\end{aligned}
$$

But $P_{A^{\dagger}}^{i} \pi_{A^{\dagger}}^{i} \tilde{\beta}=-\left(1-G_{A^{\dagger}} L\right) G_{B^{\dagger}} K=\left(G_{A^{\dagger}}-G_{B^{\dagger}}\right) K$, so

$$
\begin{aligned}
Z_{\Sigma^{\prime}}[\tilde{b}, \tilde{c}, J, K]= & \left(\operatorname{det} L_{\Sigma^{\prime}}\right)^{ \pm 1} \exp \left\{W\left((\tilde{b}-\tilde{\beta}), \sum_{i j} \phi_{1-A, A^{i j}} \tilde{c}\right)\right. \\
& -\int\left[\left(P_{A^{\dagger}}^{i} \pi_{A^{\dagger}}^{i}(\tilde{b}-\tilde{\beta}) J-\left(G_{B^{\dagger}} K\right) J\right]\right\} .
\end{aligned}
$$

Having eliminated one of the linear terms, we can now perform the integral over boundary values. (The term linear in $\tilde{b}-\widetilde{\beta}$ could also be absorbed by a shift of $\tilde{c}$, but this is not necessary; by the rules of Gaussian integration, the integral is 
now independent of this linear piece.) We find that

$$
\int_{\substack{\tilde{b}_{1}=\tilde{b}_{2} \\ \tilde{c}_{1}=\tilde{c}_{2}}}[d \tilde{b}][d \tilde{c}] Z_{\Sigma^{\prime}}[\tilde{b}, \tilde{c}, J, K]=\left(\operatorname{det} L_{\Sigma^{\prime}} \operatorname{det}\left(\sum_{i j} \phi_{1-A, A}^{i j}\right)\right)^{ \pm 1} \exp \left(\int K G_{B} J\right) .
$$

The correct Greens function for the sewn surface appears in the exponent, so the relation (3.17) between determinants is again sufficient to show that the generating functional for correlation functions sews correctly. Since any correlation function can be obtained by functionally differentiating $Z[J, K]$, this implies that arbitrary correlation functions sew correctly as well.

\section{Sewing with Zero Modes}

Our results are not yet adequate to prove sewing in string theory, since the string ghost system involves operators with zero modes. As a next generalization, we therefore consider the case in which $L$ has a kernel or cokernel.

While our results are again quite general, it is useful to keep in mind the example of the bosonic string ghosts, for which

$$
(L c)^{a b}=\nabla^{a} c^{b}+\nabla^{b} c^{a}-g^{a b} \nabla_{d} c^{d}, \quad\left(L^{\dagger} b\right)_{a}=-2 \nabla^{b} b_{a b} .
$$

For a surface of genus two or higher, $L$ has no kernel - there are no conformal Killing vectors - but it does have a cokernel, the space of holomorphic quadratic differentials. As boundary conditions, we can take those proposed by Alvarez [7], $b_{n t}=0=c^{n}$, where $n$ and $t$ denote normal and tangential components; the corresponding boundary data are $\left\{b_{n t}, c^{n}\right\}$. It is easy to check that $b_{n t}$ and $c^{n}$ are adjoint in the sense of Sect. 3. The surface term (3.8) for this example is $S_{1}=-W\left(\pi_{A^{+}} b, \pi_{1-A} c\right)=-2 \int_{C} b_{n t} c^{t}$, precisely the term proposed by Birmingham and Torre [6].

More generally, let us assume an arbitrary action of the form (3.1), but let us now allow $L_{A}$ to have a cokernel. In other words, we allow $L_{A}^{\dagger}$ to have zero modes. (The case of zero modes for $L_{A}$ can be treated similarly.) Let $Q_{A}: V^{-} \rightarrow V^{-}$ denote the orthogonal projection onto the kernel of $L_{A}^{\dagger} . L_{A}$ no longer has an inverse, but one can find a Greens function satisfying

$$
L G_{A}=1-Q_{A},\left.\quad G_{A} L\right|_{\mathrm{ker} \pi_{A}}=\left.1\right|_{\mathrm{ker} \pi_{A}}, \quad \pi_{A} G_{A}=0 .
$$

For the string ghost system, for instance, these are the standard equations for the Greens function $F_{a^{\prime} b^{\prime}}^{a}$.

An extension map $P_{A}$ satisfying (3.6) also no longer exists. Indeed, let $h \in \operatorname{ker} L_{A}^{\dagger}$; then $\int h L P_{A} \tilde{c}=W\left(\pi_{1-A^{\dagger}} h, \tilde{c}\right) \neq 0$, so we cannot require that $L P_{A}=0$. Instead, (3.6) must be replaced by

$$
\left(1-Q_{A}\right) L P_{A}=0, \quad \pi_{A} P_{A}=1,
$$

which is again satisfied by (3.7).

The extension map $P_{A^{\dagger}}$ for $b$ boundary data, in contrast, exists but is no longer unique; it is determined only up to elements of $\operatorname{ker} L_{A}^{\dagger}$. To specify $P_{A^{+}}$uniquely, 
we can require that

$$
L^{\dagger} P_{A^{\dagger}}=0, \quad \pi_{A^{\dagger}} P_{A^{\dagger}}=1, \quad Q_{A} P_{A^{\dagger}}=0 .
$$

For $A^{\dagger}$ boundary data, Eq. (3.7) must then be replaced by

$$
P_{A^{\dagger}} \pi_{A^{\dagger}}=1-G_{A^{\dagger}} L^{\dagger}-Q_{A} \text {. }
$$

As in the previous section, we can evaluate the generating functional $Z_{\Sigma^{\prime}}[\tilde{b}, \tilde{c}, J, K]$ by splitting $b$ and $c$ into classical pieces and fluctuations. We again write $c=\bar{c}+c^{\prime}$, where $\bar{c}$ is given by (5.4) and $\pi_{A} c^{\prime}=0$. For $b$, the situation is complicated by the existence of zero modes. If we let $\left\{h_{A}^{\alpha}\right\}$ denote an orthonormal basis for the kernel of $L_{A}^{\dagger}$, we can write $b=\bar{b}+b^{\prime}+\lambda_{\alpha} h_{A}^{\alpha}$, where $\bar{b}$ is given by (5.4) and $\pi_{A^{\dagger}} b^{\prime}=0=Q_{A} b^{\prime}$. For the string ghost system, for example, the $h_{A}^{\alpha}$ are the quadratic differentials satisfying Alvarez boundary conditions, and $b^{\prime}$ is a fluctuation orthogonal to these differentials. The generalization of (5.5) is then

$$
\begin{aligned}
\left(S+S_{1}+S_{2}\right)[b, c, J, K]= & S\left[b^{\prime}, c^{\prime}\right]-W\left(\pi_{A^{\dagger}} \bar{b}, \phi_{1-A, A} \pi_{A} \bar{c}\right)+\lambda_{\alpha} W\left(\pi_{1-A^{\dagger}} h_{A}^{\alpha}, \pi_{A} \bar{c}\right) \\
& +\int\left[\left(P_{A^{\dagger}} \pi_{A^{+}} \bar{b}+\lambda_{\alpha} h_{A}^{\alpha}\right) J+K\left(P_{A} \pi_{A} \bar{c}\right)-\left(G_{A^{\dagger}} K\right) J\right] .
\end{aligned}
$$

Since $b^{\prime}$ is orthogonal to the kernel of $L_{A}^{\dagger}$, the integral over $b^{\prime}$ and $c^{\prime}$ automatically gives the determinant of $L$ with the zero eigenvalues omitted. The generating functional is thus

$$
\begin{aligned}
Z_{\Sigma^{\prime}}[\tilde{b}, \tilde{c}, J, K]= & \left(\operatorname{det}^{\prime} L_{\Sigma^{\prime}}\right)^{ \pm 1} \int d \lambda_{\alpha} \exp \left\{W\left(\tilde{b}_{i}, \phi_{1-A, A}^{i j} \tilde{c}_{j}\right)-\lambda_{\alpha} W\left(\pi_{1-A^{\dagger}}^{i} h_{A}^{\alpha}, \tilde{c}_{i}\right)\right. \\
& \left.-\int\left[\left(P_{A^{\dagger}}^{i} \tilde{b}_{i}+\lambda_{\alpha} h_{A}^{\alpha}\right) J+K\left(P_{A}^{i} \pi_{A} \tilde{c}_{i}\right)-\left(G_{A^{\dagger}} K\right) J\right]\right\} .
\end{aligned}
$$

To perform the integration over boundary values required for sewing, we must again absorb the terms linear in $\tilde{c}$ by shifting $\tilde{b}$. In place of (5.7), we find that

$$
W\left(\pi_{A^{\dagger}}^{i} \beta, \phi_{1-A, A}^{i j} \pi_{A}^{j} \bar{c}\right)=-\int\left(L^{\dagger} \beta\right) P_{A}^{j} \pi_{A}^{j} \bar{c}-W\left(\pi_{1-A^{\dagger}}^{i}\left(1-Q_{A}\right) \beta, \pi_{A}^{i} \bar{c}\right) .
$$

We can therefore eliminate both terms linear in $\tilde{c}$ by choosing

$$
\beta=-G_{B^{\dagger}} K+\mu_{\alpha} h_{B}^{\alpha},
$$

where $\mu_{\alpha}$ is determined by the condition $Q_{A} \beta=\lambda_{\alpha} h_{A}^{\alpha}$. This condition will be satisfied if $M^{\alpha \beta} \mu_{\beta}=\lambda_{\alpha}+\delta_{\alpha}$, where

$$
M^{\alpha \beta}=\int h_{A}^{\alpha} h_{B}^{\beta}, \quad \delta_{\alpha}=\int h_{A}^{\alpha} G_{B^{\dagger}} K .
$$

As in the previous section, $G_{B^{\dagger}}$ is the Greens function for the sewing boundary conditions given by the projection $\pi_{B}$ of (4.8), while the $h_{B}$ are the zero modes for these boundary conditions.

Equation (6.9) can be solved for $\mu$ only if the matrix $M$ is invertible. In particular, the number of $B$ zero modes must equal the number of $A$ zero modes. We will assume for now that this is the case, and discuss the generalization in the next section. A simple calculation then shows that the generating functional is

$$
\begin{aligned}
Z_{\Sigma^{\prime}}[\tilde{b}, \tilde{c}, J, K]= & \left(\operatorname{det}^{\prime} L_{\Sigma^{\prime}}\right)^{ \pm 1} \int d\left(M^{\alpha \beta} \mu_{\beta}-\delta_{\alpha}\right) \exp \left\{W\left((\tilde{b}-\tilde{\beta}), \sum_{i j} \phi_{1-A, A}^{i j} \tilde{c}\right)\right. \\
& \left.-\int\left[\left(P_{A^{\dagger}}^{i} \pi_{A^{\dagger}}^{i}(\tilde{b}-\tilde{\beta})+\mu_{\alpha} h_{B}^{\alpha}\right) J-\left(G_{B^{\dagger}} K\right) J\right]\right\} .
\end{aligned}
$$


We can now integrate over boundary values, to find that

$$
\begin{aligned}
& \int_{\substack{\tilde{b}_{1}=\tilde{b}_{2} \\
\tilde{c}_{1}=\tilde{c}_{2}}}[d \tilde{b}][d \tilde{c}] Z_{\Sigma^{\prime}}[\tilde{b}, \tilde{c}, J, K] \\
& =\left(\operatorname{det}^{\prime} L_{\Sigma^{\prime}} \operatorname{det}\left(\sum_{i j} \phi_{1-A, A}^{i j}\right) \operatorname{det} M^{-1}\right)^{ \pm 1} \int d \mu_{\alpha} \exp \left\{\int\left[K G_{B} J-\mu_{\alpha} h_{B}^{\alpha} J\right]\right\} .
\end{aligned}
$$

The exponent is the correct one for $Z_{\Sigma}[J, K]$, and sewing will hold if

$$
\operatorname{det}^{\prime} L_{\Sigma}=\operatorname{det}^{\prime} L_{\Sigma^{\prime}} \operatorname{det}\left(\sum_{i j} \phi_{1-A, A}^{i j}\right) \operatorname{det} M^{-1},
$$

which is the generalization of (3.17) in the presence of an equal number of $A$ and $B$ zero modes.

We devote the remainder of this section to the proof of (6.13), which parallels that of (3.17) with a few added complications from the zero modes. It is somewhat easier to work with the Poisson map $\phi_{B^{\dagger}, A^{\dagger}}=\pi_{B^{\dagger}} P_{A^{\dagger}}$. Let us introduce the projection

$$
R_{B A}=h_{B}^{\alpha} M_{\alpha \beta}^{-1} h_{A}^{\beta}
$$

(that is, $\left.\left(R_{B A} b\right)(x)=h_{B}^{\alpha}(x) M_{\alpha \beta}^{-1} \int h_{A}^{\beta} b\right)$. $R$ satisfies

$$
\begin{array}{ll}
Q_{B} R_{B A}=R_{B A}, & R_{B A} Q_{B}=Q_{B}, \\
R_{B A} Q_{A}=R_{B A}, & Q_{A} R_{B A}=Q_{A} .
\end{array}
$$

In place of (4.2), the inverse of the Poisson map is now

$$
\phi_{B^{\dagger}, A^{\dagger}}^{-1}=\pi_{A^{\dagger}}\left(1-R_{B A}\right) P_{B^{\dagger}}
$$

Indeed, for the right inverse,

$$
\begin{aligned}
\phi_{B^{\dagger}, A^{\dagger}} \pi_{A^{\dagger}}\left(1-R_{B A}\right) P_{B^{\dagger}} & =\pi_{B^{\dagger}}\left(1-G_{A^{\dagger}} L^{\dagger}-Q_{A}\right)\left(1-R_{B A}\right) P_{B^{\dagger}} \\
& =\pi_{B^{\dagger}}\left(1-R_{B A}\right) P_{B^{\dagger}}=\pi_{B^{\dagger}}\left(1-Q_{B} R_{B A}\right) P_{B^{\dagger}}=1,
\end{aligned}
$$

since $\pi_{B^{\dagger}} Q_{B}=0$, while for the left in.verse

$$
\begin{aligned}
\pi_{A^{\dagger}}\left(1-R_{B A}\right) P_{B^{\dagger}} \phi_{B^{\dagger}, A^{\dagger}} & =\pi_{A^{\dagger}}\left(1-R_{B A}\right)\left(1-G_{B^{\dagger}} L^{\dagger}-Q_{B}\right) P_{A^{\dagger}} \\
& =\pi_{A^{\dagger}}\left(1-R_{B A}\right) P_{A^{\dagger}}=\pi_{A^{\dagger}}\left(1-R_{B A} Q_{A}\right) P_{A^{\dagger}}=1,
\end{aligned}
$$

since $Q_{A} P_{A^{\dagger}}=0$.

Equation (4.4) must also be modified to take the zero modes into account. It is still true that $\left(\delta L^{\dagger}\right) P_{A^{\dagger}}+L^{\dagger}\left(\delta P_{A^{\dagger}}\right)=0$, but now when we act on the left with $G_{A^{\dagger}}$ and use (6.5), we find

$$
\delta P_{A^{\dagger}}=-G_{A^{\dagger}} \delta L^{\dagger} P_{A^{\dagger}}-\left(\delta Q_{A}\right) P_{A^{\dagger}}
$$

Then in analogy to (4.6), we have

$$
\begin{aligned}
\delta \log \left(\operatorname{det} \phi_{B^{\dagger}, A^{\dagger}} \operatorname{det} L_{A^{\dagger}}^{\dagger}\right)= & \operatorname{Tr}\left[G_{A^{\dagger}} \delta L^{\dagger}-\pi_{A^{\dagger}}\left(1-R_{B A}\right) P_{B^{\dagger}} \pi_{B^{\dagger}}\left(G_{A^{\dagger}} \delta L^{\dagger}+\delta Q_{A}\right) P_{A^{\dagger}}\right] \\
= & \operatorname{Tr}\left[G_{A^{\dagger}}-P_{A^{\dagger}} \pi_{A^{\dagger}}\left(1-R_{B A}\right) P_{B^{\dagger}} \pi_{B^{\dagger}} G_{A^{\dagger}}\right] \delta L^{\dagger} \\
& -\operatorname{Tr}\left[P_{A^{\dagger}} \pi_{A^{\dagger}}\left(1-R_{B A}\right) P_{B^{\dagger}} \pi_{B^{\dagger}} \delta Q_{A}\right]
\end{aligned}
$$


Using (6.5) and (6.15), we can reduce the first term in (6.18) to

$$
\begin{aligned}
& \operatorname{Tr}\left[G_{A^{\dagger}}-\left(1-G_{A^{\dagger}} L^{\dagger}-Q_{A}\right)\left(1-R_{B A}\right)\left(1-G_{B^{\dagger}} L^{\dagger}-Q_{B}\right) G_{A^{\dagger}}\right] \delta L^{\dagger} \\
& \quad=\operatorname{Tr}\left[G_{A^{\dagger}}-\left(1-R_{B A}\right)\left(G_{A^{\dagger}}-G_{B^{\dagger}}\right)\right] \delta L^{\dagger}=\operatorname{Tr} G_{B^{\dagger}} \delta L^{\dagger}-\operatorname{Tr} G_{B^{\dagger}} \delta L^{\dagger} R_{B A},
\end{aligned}
$$

where the $R_{B A} G_{A^{\dagger}}$ term drops out because $R_{B A} G_{A^{\dagger}}=R_{B A} Q_{A} G_{A^{\dagger}}=0$. Further,

$$
-G_{B^{\dagger}} \delta L^{\dagger} R_{B A}=G_{B^{\dagger}} L^{\dagger} \delta R_{B A}=\left(1-P_{B^{\dagger}} \pi_{B^{\dagger}}-Q_{B}\right) \delta R_{B A}=\left(1-Q_{B}\right) \delta R_{B A},
$$

since $L^{\dagger} R_{B A}=0$ and $\pi_{B^{\dagger}} R_{B A}=0$.

The second term in (6.18) can also be simplified. It becomes

$$
\begin{aligned}
& -\operatorname{Tr}\left[\left(1-G_{A^{\dagger}} L^{\dagger}-Q_{A}\right)\left(1-R_{B A}\right)\left(1-G_{B^{\dagger}} L^{\dagger}-Q_{B}\right) \delta Q_{A}\right. \\
& =-\operatorname{Tr}\left[\left(1-R_{B A}\right)\left(1-G_{B^{\dagger}} L^{\dagger}\right) \delta Q_{A}\right]=-\operatorname{Tr}\left(1-R_{B A}\right) \delta Q_{A}-\operatorname{Tr}\left(1-R_{B A}\right) G_{B^{\dagger}} \delta L^{\dagger} Q_{A},
\end{aligned}
$$

and the last term vanishes by cyclicity of the trace, since $Q_{A}\left(1-R_{B A}\right)=0$. Combining these results, we have

$$
\delta \log \left(\operatorname{det} \phi_{B^{\dagger}, A^{\dagger}} \operatorname{det} L_{A^{\dagger}}^{\dagger}\right)=\delta \log \operatorname{det} L_{B^{\dagger}}^{\dagger}+\operatorname{Tr}\left[\left(1-Q_{B}\right) \delta R_{B A}-\left(1-R_{B A}\right) \delta Q_{A}\right] \text {. }
$$

To evaluate the final trace, we first observe that $\operatorname{Tr} R_{B A}$ and $\operatorname{Tr} Q_{A}$ are constants, since $R$ and $Q$ are projections. Further, $Q_{B} \delta R_{B A}=Q_{B} \delta\left(R_{B A} Q_{A}\right)=Q_{B}\left(\delta R_{B A}\right) Q_{A}+$ $R_{B A} \delta Q_{A}$. Finally, by (6.14),

$$
\operatorname{Tr} Q_{B}\left(\delta R_{B A}\right) Q_{A}=\operatorname{Tr} Q_{B}\left(h_{B}^{\alpha} \delta M_{\alpha \beta}^{-1} h_{A}^{\beta}\right) Q_{A}=\operatorname{Tr} M \delta M^{-1} .
$$

(We have used the fact that $\delta h_{A} Q_{A}=Q_{B} \delta h_{B}=0$, which follows from the orthonormality of the $h$ 's.)

Combining (6.19) and (6.20), we have

$$
\delta \log \left(\operatorname{det} \phi_{B^{\dagger}, A^{\dagger}} \operatorname{det} L_{A^{\dagger}}^{\dagger}\right)=\delta \log \operatorname{det} L_{B^{\dagger}}^{\dagger}-\delta \log \operatorname{det} M^{-1},
$$

which is the generalization of (4.7) needed to prove (6.13). Indeed, if $B$ boundary conditions are the sewing boundary conditions (4.8) of Sect. 4, we have det $\phi_{B^{\dagger}, A^{\dagger}}=$ $\operatorname{det} \sum \phi_{1-A^{\dagger}, A^{\dagger}}^{i j}$ But it is easily checked from the definition of $W$ that

$$
W\left(\phi_{1-A^{\dagger}, A^{+}} \tilde{b}, \tilde{c}\right)=-W\left(\tilde{b}, \phi_{1-A, A} \tilde{c}\right) .
$$

Hence $\operatorname{det} \sum \phi_{1-A^{\dagger}, A^{\dagger}}^{i j}=\operatorname{det} \sum \phi_{1-A, A}^{i j}$, and (6.13) follows directly.

\section{Zeros of the Poisson Map}

In the analysis of the previous section we assumed that the matrix

$$
M^{\alpha \beta}=\int h_{A}^{\alpha} h_{B}^{\beta}
$$

of inner products of the sewing zero modes $h_{B}$ with the zero modes $h_{A}$ was invertible. In particular, this requires that the number of $B$ zero modes equal the number of $A$ zero modes. For the case of the bosonic string ghosts with Alvarez boundary conditions, this is easily seen to be the case. In general, however, the matrix $M$ need not be invertible, or even square. In this section we will prove sewing with less stringent restrictions on $M$. 
We begin by recalling the generating functions (6.7) evaluated at $\tilde{b}_{1}=\tilde{b}_{2}=\tilde{b}$ and $\tilde{c}_{1}=\tilde{c}_{2}=\tilde{c}$ :

$$
\begin{aligned}
Z_{\Sigma^{\prime}}[\tilde{b}, \tilde{c}, J, K=0]= & \left(\operatorname{det} L_{\Sigma}\right)^{ \pm 1} \int d \lambda_{\alpha} \exp \left\{-S\left[\tilde{b}, \tilde{c}, \lambda_{\alpha}, J\right]\right\}, \\
S\left[\tilde{b}, \tilde{c}, \lambda_{\alpha}, J\right]= & W\left(\left(\sum_{i j} \phi^{i j}{ }_{1-A^{\dagger}, A^{\dagger}}\right) \tilde{b}+\lambda_{\alpha}\left(\pi_{1-A^{\dagger}}^{1}+\pi_{1-A^{\dagger}}^{2}\right) h_{A}^{\alpha}, \tilde{c}\right) \\
& +\int\left[\left(P_{A^{\dagger}}^{1}+P_{A^{\dagger}}^{2}\right) \tilde{b}+\lambda_{\alpha} h_{A}^{\alpha}\right] J,
\end{aligned}
$$

where we have used Eq. (6.22) to move the Poisson map from $\tilde{c}$ to $\tilde{b}$. We have also set the source $K$ which couples to the $c$ fields to zero for simplicity; the argument below can be extended to non-vanishing $K$ at the expense of a bit more algebra.

We are interested in evaluating the functional integral of $Z_{\Sigma^{\prime}}[\tilde{b}, \tilde{c}, J, K=0]$ over the boundary values $\tilde{b}$ and $\tilde{c}$. To do so, it turns out to be convenient to enlarge the space of fields slightly. We introduce additional fields $\tilde{b}^{\prime}$ and $\tilde{c}^{\prime}$ analogous to $\tilde{b}$ and $\tilde{c}$ with an action

$$
\begin{aligned}
S^{\prime}\left[\tilde{b}^{\prime}, \tilde{c}^{\prime}, \tilde{b}, \tilde{c}, J\right]= & W\left(\tilde{b}^{\prime}, \tilde{c}^{\prime}\right)+W\left(\left(\phi_{1-A^{\dagger}, A^{\dagger}}^{11}+\phi_{1-A^{\dagger}, A^{\dagger}}^{21}-\phi_{1-A^{\dagger}, A^{\dagger}}^{12}-\phi_{1-A^{\dagger}, A^{\dagger}}^{22}\right) \tilde{b}^{\prime}, \tilde{c}\right) \\
& +\int\left(P_{A^{\dagger}}^{1}-P_{A^{\dagger}}^{2}\right) \tilde{b}^{\prime} J .
\end{aligned}
$$

The field $\tilde{c}^{\prime}$ lies in the image of $\pi_{1-A}$, and the field $\tilde{b}^{\prime}$ lies in the image of $\pi_{A^{\dagger}}$. It is evident that

$$
\int\left[d \tilde{b}^{\prime}\right]\left[d \tilde{c}^{\prime}\right] \exp \left\{-S^{\prime}\left[\tilde{b}^{\prime}, \tilde{c}^{\prime}, \tilde{b}, \tilde{c}, J\right]\right\}=1 .
$$

Thus, the integral we are interested in can be written as

$$
\begin{aligned}
& \int[d \tilde{b}][d \tilde{c}] Z_{\Sigma^{\prime}}[\tilde{b}, \tilde{c}, J, K=0] \\
& \quad=\int\left[d \tilde{b}^{\prime}\right]\left[d \tilde{c}^{\prime}\right][d \tilde{b}][d \tilde{c}] d \lambda_{\alpha} \exp \left\{-\left(S+S^{\prime}\right)\left[\tilde{b}, \tilde{c}, \tilde{b}^{\prime}, \tilde{c}^{\prime}, \lambda_{\alpha}, J\right]\right\} .
\end{aligned}
$$

The reason for the apparently ad hoc introduction of the fields $\tilde{b}^{\prime}$ and $\tilde{c}^{\prime}$ becomes clear upon changing variables in the right-hand side of (7.5) to

$$
\begin{array}{ll}
\mathbf{b}_{1}=\tilde{b}+\tilde{b}^{\prime} & \mathbf{b}_{2}=\tilde{b}-\tilde{b}^{\prime}, \\
\mathbf{c}_{\mathbf{1}}=\frac{1}{2} \tilde{c}^{\prime}, & \mathbf{c}_{\mathbf{2}}=\tilde{c} .
\end{array}
$$

In terms of the fields $\mathbf{b}_{i}$ and $\mathbf{c}_{i}$ the action $S+S^{\prime}$ takes the form

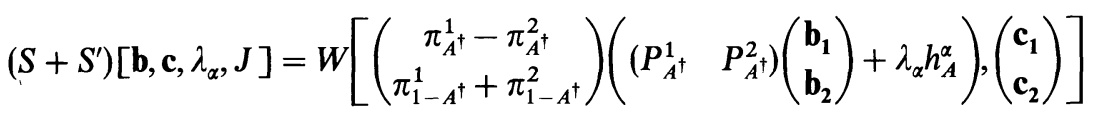

$$
\begin{aligned}
& \left.+\int\left(\begin{array}{ll}
\left(P_{A^{\dagger}}^{1}\right. & P_{A^{\dagger}}^{2}
\end{array}\right)\left(\begin{array}{l}
\mathbf{b}_{1} \\
\mathbf{b}_{2}
\end{array}\right)+\lambda_{\alpha} h_{A}^{\alpha}\right) J .
\end{aligned}
$$

Here we have used $\pi_{A^{\dagger}}^{1} h_{A}=\pi_{A^{\dagger}}^{2} h_{A}=0$. In this expression we recognize the projection $\pi_{B^{\dagger}}=\left(\begin{array}{c}\pi_{A^{\dagger}}^{1}-\pi_{A^{\dagger}}^{2} \\ \pi_{1-A^{\dagger}}^{1}+\pi_{1^{-} A^{\dagger}}^{2}\end{array}\right)$ of (4.8) and the extension map $P_{A^{\dagger}}=\left(P_{A^{\dagger}}^{1} P_{A^{\dagger}}^{2}\right)$ of (3.14). The integral thus takes the compact form 


$$
\begin{aligned}
\int[d \tilde{b}][d \tilde{c}] Z_{\Sigma^{\prime}}[\tilde{b}, \tilde{c}, J] & =\int[d \mathbf{b}][d \mathbf{c}] d \lambda_{\alpha} \exp \left\{-S^{\prime \prime}\left[\mathbf{b}, \mathbf{c}, \lambda_{\alpha}, J\right]\right\}, \\
S^{\prime \prime}\left[\mathbf{b}, \mathbf{c}, \lambda_{\alpha}, J\right] & =W\left(\pi_{B^{+}}\left(P_{A^{+}} \mathbf{b}+\lambda_{\alpha} h_{A}^{\alpha}\right), \mathbf{c}\right)+\int\left(P_{A^{\dagger}} \mathbf{b}+\lambda_{\alpha} h_{A}^{\alpha}\right) J,
\end{aligned}
$$

with

$$
\mathbf{b}=\left(\begin{array}{l}
\mathbf{b}_{1} \\
\mathbf{b}_{2}
\end{array}\right), \quad \mathbf{c}=\left(\begin{array}{l}
\mathbf{c}_{1} \\
\mathbf{c}_{2}
\end{array}\right) .
$$

We now turn to the evaluation of (7.8). The integration over $\mathbf{b}$ and $\mathbf{c}$ can be performed by shifting $\mathbf{b}$ and $\mathbf{c}$ so as to eliminate the linear terms. This clearly requires a Greens function for the Poisson map $\phi_{B^{\dagger}, A^{\dagger}}=\pi_{B^{\dagger}} P_{A^{\dagger}}$. Our previous assumption of an invertible matrix $M$ of inner products between the $A$ zero modes and the $B$ zero modes was equivalent to the assumption that $\phi_{B^{\dagger}, A^{\dagger}}$ was invertible; in fact, we wrote down the explicit inverse in (6.16). Let us now make the weaker assumption that $\phi_{B^{\dagger} A^{\dagger}}$ has a kernel, but no cokernel. Equivalently we assume that the matrix $M$ of (7.1) has a right inverse, but no left inverse. There is thus a Green's function $G_{\phi}$ for $\phi_{B^{\dagger}, A^{\dagger}}$ satisfying

$$
\phi_{B^{\dagger}, A^{\dagger}} G_{\phi}=1, \quad G_{\phi} \phi_{B^{\dagger}, A^{\dagger}}=1-Q_{\phi},
$$

where $Q_{\phi}$ is the orthogonal projection onto the zero modes of $\phi_{B^{\dagger}, A^{\dagger}}$.

If we now shift the fields by

$$
\mathbf{c} \mapsto \mathbf{c}+G_{\phi} P_{A^{\dagger}}^{\dagger} J, \quad \mathbf{b} \mapsto \mathbf{b}+G_{\phi} \pi_{B^{\dagger}} h_{A},
$$

a little algebra then shows that

$$
\left(S+S^{\prime}\right)\left[\mathbf{b}, \mathbf{c}, \lambda_{\alpha}, J\right] \mapsto W\left(\phi_{B^{\dagger}, A^{\dagger}}\left(1-Q_{\phi}\right) \mathbf{b}, \mathbf{c}\right)+\int S_{B \phi} Q_{\phi} \mathbf{b} J+\lambda_{\alpha} \int R_{B A} h_{A}^{\alpha} J
$$

with

$$
R_{B A}=\left(1-P_{A^{\dagger}} G_{\phi} \pi_{B^{\dagger}}\right) Q_{A}, \quad S_{B \phi}=P_{A^{\dagger}} Q_{\phi} .
$$

Let us consider the maps $R_{B A}$ and $S_{B \phi}$ more carefully. It is easy to check that $R_{B A}$ is a projection satisfying

$$
Q_{B} R_{B A}=R_{B A}, \quad R_{B A} Q_{A}=R_{B A} .
$$

In particular, $R_{B A}$ maps the kernel of $L_{A^{\dagger}}^{\dagger}$ into the kernel of $L_{B^{\dagger}}^{\dagger}$. The projection $R_{B A}$ also satisfies $Q_{A} R_{B A}=Q_{A}$. Thus $R_{B A}$ satisfies all the conditions (6.15) of the projection $R_{B A}$ of the previous section, except that now $R_{B A} Q_{B} \neq Q_{B}$.

The map $S_{B \phi}$ satisfies

$$
S_{B \phi} Q_{\phi}=S_{B \phi}, \quad Q_{B} S_{B \phi}=S_{B \phi} .
$$

The first of these equations is obvious, while the second is a consequence of the facts that

$$
\pi_{B^{\dagger}} S_{B \phi}=\pi_{B^{\dagger}} P_{A^{\dagger}} Q_{\phi}=\phi_{B^{\dagger}, A^{\dagger}} Q_{\phi}=0, \quad L^{\dagger} S_{B \phi}=L^{\dagger} P_{A^{\dagger}} Q_{\phi}=0 .
$$

Equation (7.15) means in particular that $S_{B \phi}$ maps the kernel of $\phi_{B^{\dagger}, A^{\dagger}}$ into the kernel of $L_{B^{\dagger}}^{\dagger} S_{B \phi}$ also satisfies $Q_{A} S_{B \phi}=0$ (since $Q_{A} P_{A^{\dagger}}=0$ ).

Let us now define 


$$
\mathscr{R}_{B A}=\left(R_{B A} \quad S_{B \phi}\right): \operatorname{ker} L_{A^{\dagger}}^{\dagger} \oplus \operatorname{ker} \phi_{B^{\dagger}, A^{\dagger}} \rightarrow \operatorname{ker} L_{B^{\dagger}}^{\dagger}
$$

It may be checked that $\mathscr{R}_{B A}$ is invertible, with

$$
\mathscr{R}_{B A}^{-1}=\left(\begin{array}{c}
Q_{A} Q_{B} \\
Q_{\phi} \pi_{A^{\dagger}} Q_{B}
\end{array}\right) .
$$

In particular, this means that

$$
\operatorname{dim} \operatorname{ker} L_{A^{\dagger}}^{\dagger}+\operatorname{dim} \operatorname{ker} \phi_{B^{\dagger}, A^{\dagger}}=\operatorname{dim} \operatorname{ker} L_{B^{\dagger}}^{\dagger}
$$

That is, the number of zero modes of $\phi_{B^{\dagger}, A^{\dagger}}$ is the difference between the number of zero modes of $L_{B^{\dagger}}^{\dagger}$ and those of $L_{A^{\dagger}}^{\dagger}$.

Using $\mathscr{R}_{B A}$, we can now easily give the result of integrating $Z_{\Sigma^{\prime}}$ over the boundary values $\tilde{b}$ and $\tilde{c}$. We find

$$
\int[d \tilde{b}][d \tilde{c}] Z_{\Sigma^{\prime}}[\tilde{b}, \tilde{c}, J, K=0]=\left(\operatorname{det}^{\prime} L_{A^{\dagger}}^{\dagger} \operatorname{det}^{\prime} \phi_{B^{\dagger}, A^{\dagger}} \operatorname{det} \mathscr{R}_{B A}\right)^{ \pm} \int d \mu_{\alpha} \exp \left\{-\int \mu_{\alpha} h_{B}^{\alpha} J\right\} .
$$

The $\mu$ integral is the correct one for $Z_{\Sigma}[J, K=0]$, so sewing will hold if

$$
\operatorname{det}^{\prime} L_{, A^{\dagger}}^{\dagger}=\operatorname{det}^{\prime} L_{A^{\dagger}}^{\dagger} \operatorname{det}^{\prime} \phi_{B^{\dagger}, A^{\dagger}} \operatorname{det} \mathscr{R}_{B A} \text {. }
$$

This is the generalization of (6.13) in the presence of more zero modes for $B$ than for $A$.

The proof that (7.21) is valid is similar to the proof of (6.16), and involves verifying the infinitesimal relation

$$
\operatorname{Tr} \mathscr{R}_{B \boldsymbol{A}}^{-1} \delta \mathscr{R}_{B \boldsymbol{A}}=\operatorname{Tr} G_{B^{\dagger}} \delta L^{\dagger}-\operatorname{Tr} G_{A^{\dagger}} \delta L^{\dagger}-\operatorname{Tr} G_{\phi} \delta \phi_{B^{\dagger}, A^{\dagger}}
$$

We omit the details.

Let us now briefly discuss the geometric interpretation of the determinant formula (7.21). For this, we need some notions from the theory of determinant line bundles (see [8]). Let $V^{ \pm}$be Hilbert spaces and $D_{p}: V^{+} \rightarrow V^{-}, p \in \mathscr{P}$, be a family of linear operators depending smoothly on a parameter space $\mathscr{P}$. Under suitable conditions [8], the family $D$ determines a natural line bundle Det $D$ over $\mathscr{P}$ whose fiber at $p$ is canonically isomorphic to

$$
(\operatorname{Det} D)_{p} \equiv\left(\operatorname{Det} \operatorname{ker} D_{p}\right)^{-1} \otimes \operatorname{Det} \operatorname{ker} D_{p}^{\dagger} \text {. }
$$

Here $\operatorname{Det}(X)$ denotes the highest exterior power of the finite dimensional vector space $X$, and $X^{-1}$ the dual space of $X$.

The bundle Det $D$ is called the determinant line bundle. It comes equipped with a natural covariant derivative defined as follows. We consider $V^{ \pm} \times \mathscr{P}$ as trivial bundles over $\mathscr{P}$ with trivial flat covariant derivatives. These allow us to define the covariant derivative $\nabla D$ of $D$ as a map from $V^{+}$to $V^{-}$. Moreover, as sub-bundles of $V^{ \pm} \times \mathscr{P}$, the bundles $\operatorname{ker} D$ and $\operatorname{ker} D^{\dagger}$ inherit covariant derivatives, and by linear algebra there are induced covariant derivatives on $\operatorname{Det} \operatorname{ker} D$ and $\operatorname{Det} \operatorname{ker} D^{\dagger}$. The covariant derivative on Det $D$ is then defined as

$$
\nabla_{\text {Det } D}=-\nabla_{\text {Det ker } D}+\nabla_{\text {Det ker } D^{\dagger}}+\operatorname{Tr}_{\text {ker } D^{\perp}} D^{-1} \nabla D,
$$

where $\operatorname{Tr}_{\text {ker } D^{\perp}}$ denotes the trace restricted to the orthogonal complement of $\operatorname{ker} D$. 
Since Det $D$ is a line bundle, it has a unique inner product compatible with $\nabla_{\text {Det } D}$. This is given by

$$
\text { | }\left.\right|_{\operatorname{Det} D}=\left(||_{\operatorname{Det} k e r} D^{-1}\left(|\quad|_{\operatorname{Det} \operatorname{ker} D^{\dagger}}\right) \operatorname{det}^{\prime} D^{\dagger} D,\right.
$$

where ||$_{\text {Det ker } D}$ and ||$_{\text {Det ker } D^{\dagger}}$ are the inner products on Det $\operatorname{ker} D$ and Det $\operatorname{ker} D^{\dagger}$ induced from those on $V^{ \pm}$.

With these notions understood, we may now describe the geometric meaning of Eq. (7.21). By linear algebra, the isomorphism $\mathscr{R}_{B A}$ gives an isomorphism

$$
\text { Det } \mathscr{R}_{B A} \text { : Det ker } L_{A^{\dagger}}^{\dagger} \otimes \operatorname{Det} \operatorname{ker} \phi_{B^{\dagger}, A^{\dagger}} \rightarrow \operatorname{Det} \operatorname{ker} L_{B^{\dagger}}^{\dagger} \text {. }
$$

Since $\operatorname{ker} L_{A}, \operatorname{ker} L_{B}$ and $\operatorname{ker} \phi_{B A}$ are all empty, this may be considered as a map

$$
\operatorname{Det} \mathscr{R}_{B A}: \operatorname{Det} L_{A} \otimes \operatorname{Det} \phi_{B A} \rightarrow \operatorname{Det} L_{B} \text {. }
$$

Suppose now that the operators $L$ depend smoothly on some parameter space $\mathscr{P}$. If the determinant lines Det $L_{A}$, Det $L_{B}$ and Det $\phi_{B A}$ over $\mathscr{P}$ are given the natural covariant derivatives (7.24), the determinant formulas in the infinitesimal form (7.22) and the integrated form (7.21) imply respectively

$$
\nabla \operatorname{Det} \mathscr{R}_{B A}=0, \quad\left|\operatorname{Det} \mathscr{R}_{B A}\right|=\text { constant. }
$$

Det $\mathscr{R}_{\boldsymbol{B A}}$ is thus a natural geometric quantity: it is a covariantly constant trivialization of the line Det $L_{A} \otimes \operatorname{Det} \phi_{B A} \otimes\left(\operatorname{Det} L_{B}\right)^{-1}$.

\section{Conclusion}

We have now demonstrated quite generally that Gaussian conformal field theories "sew," that is, that correlation functions on a surface $\Sigma$ can be obtained from correlation functions on a corresponding cut surfaces $\Sigma^{\prime}$ by integrating over appropriate boundary data. Our proof does not apply directly to more complicated theories with nonquadratic interactions, for which correlation functions have no simple expression in terms of determinants. However, correlation functions for such theories can be expressed perturbatively in terms of correlation functions for Gaussian theories; our results thus apply at least perturbatively to any conformal field theory which can be derived from a path integral.

Several straightforward generalizations of this work are possible. For simplicity, we restricted our attention in this paper to sewn surfaces $\Sigma$ with no remaining unsewn boundaries, but the same methods can be used to show that sewing also holds when $\Sigma$ has boundaries. Further, although our examples were all twodimensional, our proofs made no use of that fact; our techniques can be used to demonstrate sewing in arbitrary dimensions.

As a further extension, we may consider a sewing problem in which different choices of boundary data, say $A$ and $A^{\prime}$, are made for the two boundaries being sewn. Such a situation naturally arises when one imposes APS-type boundary conditions [9], in which positive frequency components of fields are specified at the boundary: one should then equate positive frequency (outgoing) data at one boundary with negative frequency (incoming) data at the other. This type of problem is most easily handled by first sewing to one boundary an infinitesimal cylinder 
with $A$ boundary data on one end and $A^{\prime}$ boundary data on the other, thus reducing the problem to the type already considered. We can then make contact with the operator formalism of Alvarez-Gaumé et al. [10]: the positive frequency components of fields on a circle $|z|=1$ can be extended to $z=0$ to give data corresponding to a punctured surface, and our intermediate infinitesimal cylinder corresponds to their "sewing state" $|S\rangle$.

To apply these results to string theory, we must still understand how to construct the moduli space for the sewn surface $\Sigma$ from the moduli space for the cut surface $\Sigma^{\prime}$. This will be the subject of a future paper.

Acknowledgements. We would like to thank Steven Blau for valuable discussions during the early stages of this work.

\section{References}

1. Tseytlin, A. A.: Phys. Lett 168B, 63 (1986)

2. Sonoda, H.: Lawrence Berkeley Laboratory preprints LBL-25140 and LBL-25316, 1988

3. Carlip, S.: Phys. Lett 209B, 464 (1988)

4. DeWitt, B. S.: In: Relativity, groups and topology II. Amsterdam: North-Holland 1984

5. Forman, R.: Invent. Math. 88, 477 (1987)

6. Birmingham, D., Torre, C. G.: Class. Quant. Grav. 4, 1149 (1987)

7. Alvarez, A.: Nucl. Phys. B216, 125 (1983)

8. Bismut, J. M., Freed, D.: Commun. Math. Phys. 106, 159 (1986)

9. Atiyah, M. F., Patodi, V. K., Singer, I. M.: Math. Proc. Camb. Phil. Soc. 77, 43 (1975)

10. Alvarez-Gaumé, L., Gomez, C., Moore, G., Vafa, C.: Nucl. Phys. B303, 455 (1988)

Communicated by L. Alvarez-Gaumé

Received February 27, 1989 
